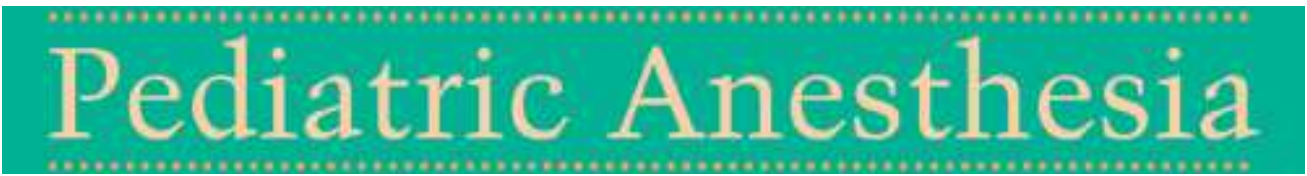

\title{
Cannot ventilate-cannot intubate an infant: Surgical tracheotomy or transtracheal cannula?
}

\begin{tabular}{|r|l|}
\hline Journal: & Pediatric Anesthesia \\
\hline Manuscript ID: & PAN-2010-0263.R1 \\
\hline Manuscript Type: & Original Paper \\
\hline Author: & 05-Jul-2010 \\
\hline Complete List of Authors: & $\begin{array}{l}\text { Johansen, Karina; Rigshospitalet, Anaesthesia, 4231 } \\
\text { Holm-Knudsen, Rolf; Rigshospitalet, Anaesthesia, 4231 } \\
\text { Charabi, Birgitte; Righospitalet, Head and Neck Surgery } \\
\text { Kristensen, Michael; Rigshospitalet, Anaesthesia, 4231 } \\
\text { Rasmussen, Lars; Rigshospitalet, Anaesthesia, 4231 }\end{array}$ \\
\hline Key Words: & $\begin{array}{l}\text { difficult airway, animal model, airway algorithm, emergency } \\
\text { tracheotomy, children, transtracheal cannula }\end{array}$ \\
\hline
\end{tabular}

\section{S) ScholarONE




\section{Cannot ventilate-cannot intubate an infant: \\ Surgical tracheotomy or transtracheal cannula?}

Karina Johansen ${ }^{1}$, Rolf J. Holm-Knudsen ${ }^{1}$, Birgitte Charabi $^{2}$, Michael Seltz Kristensen ${ }^{1}$, Lars Simon Rasmussen".

1 Department of Anaesthesia, Centre of Head and Orthopaedics,

Rigshospitalet, Copenhagen University Hospital, Copenhagen, Denmark.

2 Department of ORL, Head and Neck Surgery, Centre of Head and Orthopedics, Rigshospitalet, Copenhagen University Hospital, Copenhagen, Denmark.

Running head: Surgical tracheotomy or transtracheal cannula in infants?

Correspondance:

Karina Johansen, Department of Anaesthesia 4231, Rigshospitalet, Blegdamsvej 9, DK 2100 Copenhagen, Denmark

Email: karinakjohansen@hotmail.com 


\section{Summary}

Background: An unanticipated difficult airway is very uncommon in infants.

The recommendations for managing the can't ventilate-can't intubate ('CVCl') situation in infants and small children are based on difficult airway algorithms for adults. These algorithms usually recommend placement of a transtracheal cannula or performing a surgical tracheotomy as a last resort. In this study we compared the success rate and time used for inserting a transtracheal cannula versus performing a modified surgical tracheotomy in a piglet model.

Methods: We used 10 three-weeks-old euthanized piglets, weighing eight kilograms each. Thirty physicians had a timed attempt of inserting a transtracheal cannula for jet ventilation. A maximum time of $120 \mathrm{sec}$ was allowed. Ten physicians had a timed attempt of performing a modified surgical tracheotomy after a short introduction by an ENT surgeon. The allowed time for this procedure was $240 \mathrm{sec}$.

Results: Placement of the transtracheal cannula was successful for eight of 30 physicians. Median time for successful insertion was $68 \mathrm{sec}$. Surgical tracheotomy was successful for eight of 10 physicians. Median time for successful tracheotomy was $89 \mathrm{sec}$. There was a significantly higher success rate for surgical tracheotomy $(P=0.007)$.

Conclusions: We found placement of a transtracheal cannula to be significantly less successful than the modified surgical tracheotomy in a piglet 
model. We question if placement of a transtracheal cannula should be recommended in infants in a can't ventilate-can't intubate situation.

Keywords: difficult airway; animal model; airway algorithm; emergency tracheotomy; children; transtracheal cannula. 


\section{Introduction}

In healthy infants an unanticipated difficult airway is very uncommon. The horrifying situation in which ventilation, oxygenation, and intubation are impossible, is fortunately extremely rare and most anaesthetists will rarely - if ever - encounter this scenario. In this situation when all other means have failed, an invasive technique to ensure oxygenation must be considered. In adult airway algorithms, it is usually recommended to do a needle or surgical cricothyroidotomy, but in infants or small children the recommendations are less clear $(1,2)$. Only one national recommendation exists for children and the last step in the 'can't ventilate-can't intubate' situation ('CVCl') is "a rapid tracheal access or transtracheal jet ventilation" (3). The technique to achieve this is not specified.

As the situation is extremely rare, no comparative studies exist and probably very few anaesthetists have any experience in invasive techniques that allow emergency airway access in small children thus a suitable training model of this situation is both necessary and difficult to make. Practicing on a manikin may help in decision-making and team work, but it is far from reality to palpate and place a needle on a silicone manikin. An animal model will be more realistic, but only one infant model using kittens has been presented in the literature (4).

The aim of this study was to compare the success rate and time used for placing a transtracheal cannula versus performing a modified surgical tracheotomy in a piglet model. 


\section{Materials and methods}

Piglets were chosen for the model as the authors have a broad experience in using pig necks as an adult model for practicing emergency airway access. The airway of eight kilogram piglets have dimensions comparable to small children. When the piglets are placed on their backs, the trachea and larynx are identified as soft structures as in children.

Ten three-weeks-old piglets, weighing eight kilograms were euthanized by a veterinarian.

Thirty physicians who attended a difficult airway workshop, agreed to participate after being informed about the study.

The participants had prior to the workshop attended lectures on transtracheal jet ventilation and paediatric emergency tracheotomy. During the workshop an ENT surgeon introduced the participants to the tracheotomy technique and the equipment to be used.

For the transtracheal cannula placement the technique recommended by The European Resuscitation Council guidelines was used (5). A water filled $10 \mathrm{ml}$ syringe with a standard 16 or $18 \mathrm{GA}$ intravenous cannula (Venflon ${ }^{\mathrm{TM}}$ Pro) attached was used to puncture the trachea just inferior to the cricoid cartilage percutaneously at an angle of $45^{\circ}$ caudally (Figure 1). After verification of correct position by air aspiration, the needle was removed and the cannula was connected via a three-way stopcock to a high-pressure oxygen source. Inspiration or "jetting" was performed by occluding the free limb of the 
stopcock by a finger causing oxygen to be insufflated via the cannula for one second followed by four sec for "expiration" (Figure 2).

The participants were timed from the start of palpation on the piglets' neck. Jet ventilation was started when the participant was convinced that the cannula was placed in the trachea. Verification of cannula placement was performed clinically by watching movement of the thorax. If in doubt, the airflow was increased and/or the escape of air prevented via the upper airway. An attempt was considered a failure if a functioning cannula was not in place within $120 \mathrm{sec}$ from start, or if "jetting" was attempted with a non-functioning cannula. The time limit was set to make it more "realistic": If the trachea is not cannulated within two minutes of starting the procedure the hypoxia will be life-threatening. In addition, injury to several adjacent structures would likely have occurred, why it would be meaningless to pursue the same strategy.

For the modified surgical tracheotomy we selected tools readily available in theatre. A scalpel, a pair of scissors with a sharp tip and three towel forceps (Figure 3). The larynx and proximal trachea were identified by palpation, and a vertical incision was performed through the skin and subcutaneous tissue from the upper part of the larynx to the sternal notch. The strap muscles were grasped with two towel forceps and separated in the midline giving access to palpate and identify the trachea. Bleeding makes visual identification of the trachea more difficult and the participants were encouraged not to look for but to palpate the tracheal rings in order to indentify the trachea. The trachea was 
stabilised by grasping it from side to side with a towel forceps, and while the sharp tip of the scissors was inserted between two tracheal rings one to two cm distal to the larynx, the trachea was lifted anteriorly in order not to damage the posterior wall or resect the cartilage (Figure 4). With the scissors a vertical cut in the midline of the trachea in the distal direction was made, and the endotracheal tube was inserted. Placement was verified by successful ventilation of the lungs or by fibroscopy.

A tracheotomy attempt was considered a failure if no endotracheal tube was placed within $240 \mathrm{sec}$ since this procedure would result in a definitive airway. If more than four minutes are used for the emergency tracheotomy a good outcome will be less likely. First all the participants, three per piglet, had a timed attempt of placing a transtracheal cannula for jet ventilation. Ten randomly selected participants subsequently had a timed attempt of doing a modified surgical tracheotomy and inserting a cuffed endotracheal tube size $3.5 \mathrm{~mm}$ ID.

The participants were told about the maximum time allowed prior to the study.

Before starting the procedure, in half of the piglets the tip of an Olympus Lf-2 flexible fiberscope, connected to an Olympus CLE cold light source was placed at the level of the vocal cords by means of direct laryngoscopy. In this way the subglottic space and the trachea were observed during the entire procedure. The observations obtained via the fiberscope were not available to the person performing the transtracheal procedure nor to the person doing the timing and clinical confirmation of the cannula placement. 
After the workshop the trachea of the piglets were transected just below the cricoid cartilage and the tracheal diameter was measured.

\section{Statistics}

Continuous data are reported with median and range, and proportions are reported with $95 \%$ confidence intervals.

The primary end point was the time for obtaining access to the airway, either by insertion of the transtracheal cannula or by performing surgical tracheotomy. This was compared using the Mann-Whitney test as we considered the attempts to be independent. The secondary end point was the success rate for the procedure. This was compared with Fisher's Exact test, based on the assumption that the two procedures were independent. We illustrated the time and success using Kaplan-Meier statistics. A p-value less than 0.05 was considered significant.

\section{Results}

Introduction of the flexible scope allowed adequate visualization of the subglottic space and the trachea during the entire procedure in the five piglets, in which this was attempted.

Placement of a transtracheal cannula was successful in eight of 30 (27\%) attempts within $120 \mathrm{sec}$. The median time of the eight successful insertions was $68 \mathrm{sec}(35-95 \mathrm{sec})$ until jet ventilation was established. Visualization using the fiberscope was carried out in 14 of these 30 attempts. The examination revealed a discrepancy between the clinical verification of the cannula placement and the actual placement in four of the $14(29 \%)$ 
attempts. This included attempted jet ventilation despite paratracheal placement, a kinked catheter, and also a correctly placed cannula, but no jet ventilation because of uncertainty of the cannula placement.

A modified surgical tracheotomy was performed successfully in eight of 10 (80\%) participants within $240 \mathrm{sec}$. The median time for successful insertion of the endotracheal tube with this procedure was $89 \mathrm{sec}(71-200 \mathrm{sec})$. The ten participants performing a tracheotomy had a similar success rate of transtracheal cannula placement as the other participants (two out of 10).

The successful placement of a transtracheal cannula tended to be faster $(P=0.08)$ (Table 1, Figure 5), but the success rate for surgical tracheotomy was significantly higher $(P=0.007)$.

The fiberoptic examination was performed in five of the 10 attempts of surgical tracheotomy and confirmed correct placement of the endotracheal tube in all five cases. The tracheal diameter was eight $\mathrm{mm}(6-9 \mathrm{~mm})$

\section{Discussion}

We found that anaesthetists had significantly less success in placing a transtracheal cannula within $120 \mathrm{sec}$ than in performing a modified surgical tracheotomy within $240 \mathrm{sec}$.

In infants and small children the dimensions of the cricothyroid membrane are small and the cricoid cartilage is narrow, which makes it difficult or impossible to pass an endotracheal tube through the membrane without risk of fracturing the cartilages of the larynx (6). This combined with the difficulty in identifying the membrane in infants because of the short neck, the cephalad position of 
larynx, the softness of the cartilages, and the prominent hyoid bone, which is easily mistaken for the thyroid cartilage, makes it unattractive to perform cricothyroidotomy in small children (7).

The alternatives left in a 'CVCl' situation are to perform an emergency tracheotomy or insert a transtracheal cannula. The tracheotomy is a definitive airway, which can be used for ventilation, whereas the transtracheal cannula only is a temporary measure, which makes it possible to oxygenate the child with modified jet ventilation until surgical expertise arrives to perform a tracheotomy. Despite this, since anaesthetists are very familiar with cannula techniques and not surgically trained, the transtracheal cannula insertion will be the most appealing choice to the majority of anaesthetists.

In our study surprisingly many of the participants had difficulty inserting a transtracheal cannula in the piglet model, despite the relative ease of palpating the larynx and upper part of the trachea. Only $27 \%$ succeeded within the time limit of $120 \mathrm{sec}$. In the 14 attempts assessed via the endoscope we also noticed that the anaesthetist had difficulty determining whether the cannula was in the tracheal lumen or not. In one case the cannula was correctly placed but kinked and could not be used. This could probably have been avoided by using a more kink resistant device as for instance the "Ravussin catheter". The success rate of transtracheal cannula was low and there is a risk of serious complications such as air embolism, extensive cervical emphysema, pneumothorax and 
pneumomediastinum, and damage to the oesophagus if jet ventilation is attempted using a misplaced cannula.

We found that a short introduction to a modified surgical tracheotomy proved to be sufficient to make eight of 10 participants able to perform successfully on the model within the predefined time limit. The median time for successful tracheotomy was $89 \mathrm{sec}$. Although this is $21 \mathrm{sec}$ more than for the successful insertion of a transtracheal cannula, the success rate was three times higher. In addition, the tracheotomy is a secure airway, whereas the transtracheal cannula is only a temporary airway, which easily displaces and must be substituted urgently with another one.

The tools available were chosen to be as simple and few as possible to simulate a realistic scenario. A scalpel is always readily available in theatre and a pair of scissors and the towel forceps are standard equipment in our anaesthetic trolley. We chose to make the tracheal incision with the scissors instead of using the scalpel, to make it easier for a surgically untrained person to control the size of the tracheal incision.

This piglet model of course has limitations. It is not possible to create the same extreme level of stress that a severely hypoxic child will cause. But the stress will probably be equally disadvantageous in both procedures. As the piglets were dead, there was no bleeding during the procedure. Bleeding must be expected in a real situation and will add to the stress and make the procedures more difficult. In order to compensate for this we encouraged the participant not to depend on vision during identification of the trachea and 
instead palpate to find the tracheal rings. The strengths of this model are that the palpation for the landmarks and the sensations during cannulation and surgery are very realistic and much better than when using manikins. The piglets size corresponds to an infant of 6 months to 3 years.

In adults a transtracheal cannula is a well-established way of keeping an acceptable oxygenation during a 'CVCl' situation until a more secure airway is established $(8,9)$. This is probably the explanation for recommending this procedure as a last resort in the emergency airway management in infants and small children as well $(6,7)(10,11)$.

The evidence for this recommendation does nevertheless not exist. Only a few papers, including one case report and one small series of elective percutaneous transtracheal jet ventilation, using the specially designed “Ravussin catheter", in children have been published $(13,14)$. The transtracheal cannulas were placed during controlled circumstances under direct endoscopic visual guidance in a well-oxygenated child. Despite this, serious complications occurred, including bilateral pneumothorax. This situation is very different form the chaotic situation that rules at the time point, when the anaesthetist finally as the last resort decides to attempt placing a transtracheal cannula in a severely hypoxic child.

In the literature we have not found any studies documenting success or risks associated with needle cricothyroidotomy or transtracheal needle access in a 'CVCl' situation in infants. Only one case with a successful placement of a 
transtracheal cannula in a six year old, $20 \mathrm{~kg}$ Treacher Collins girl was found. In this case the cannula was placed via a pre-existing tracheal fistula (15).

Currently the recommendations are based on expert's opinion and reviews of articles focused on adult airway management (8) (10). Weiss et al (16) recently proposed an algorithm for the management of the unexpected difficult paediatric airway based on the Difficult Airway Society's (DAS) recommendations for adults (1). As their rescue technique in the 'CVCl' situation they reserve cannula cricothyroidotomy for children older than eight, but they recommend surgical cricothyroidotomy in patients of all ages.

The 'CVCl' situation in a small child without anticipated difficult airway is extremely rare. As with other rare situations, ie malignant hyperthermia, it is important to be prepared and practice the situation on models or in simulators to improve the chance of survival of the patient. Not only the technical skills but also decision making should be trained.

Thorough preoperative assessment, clinical education, simulator training, and implementation of national society recommendations are all important in order to reduce the occurrence of a failed airway (16). We think this should be combined with further development of an animal model for training and practicing surgical emergency airway access in infants and children. The setup described by Heard et al. (8) seems ideal, but the use of live animals is very demanding and costly and therefore probably not an option in most departments. 
We cannot draw any firm conclusions from this small study, but it may stimulate to some thoughts and more studies, involving animal models, on emergency airways in small children. We found the placement of a transtracheal needle to be more difficult and less successful than the modified surgical tracheotomy in a piglet model. We question if placement of a transtracheal cannula should be recommended in infants in a can't ventilatecan't intubate situation. 
References

1 Henderson JJ, MT Popat, Latto IP et al. Difficult Airway Society guidelines for management of the unanticipated difficult intubation. Anaesthesia 2004; 59: 675-694.

2 Practice Guidelines for Management of the Difficult Airway. An Updated report by the American Society of Anesthesiologists Task Force on Management of the Difficult Airway. Anesthesiology 2003; 98: 1269-1277.

3 Frova G, Guarino A, Petrini F et al. Recommendations for airway control and difficult airway management in paediatric patients. Minerva Anestesiol 2006; 72: 723-748.

4 McLaughlin J, Iserson KV. Emergency pediatric tracheostomy: a usable technique and model for instruction. Ann Emerg Med 1986; 15: 463-465.

5 European Resuscitation Council. Airway and ventilation. In: European Paediatric Life Support, Course Manual, 3rd edition, Antwerp: European Resuscitation Council Secretariat VZW, 2006: 41-42.

6 Navsa N, Tossel G, Boon JM. Dimensions of the neonatal cricothyroid membrane - how feasible is a surgical cricothyroidotomy? Pediatr Anesth 2005; 15: 402-406.

7 Cote CJ, Hartnick CJ. Pediatric transtracheal and cricothyrotomy airway devices for emergency use: which are appropriate for infants and children? Pediatr Anesth 2009; 19: 66-76.

8 Heard AM, Green RJ, Eakins P. The formulation and introduction of a 'can't intubate, can't ventilate' algorithm into clinical practice. Anaesthesia 2009; 64: 601-608. 
9 Benumof JL, Scheller MS. The importance of transtracheal jet ventilation in the management of the difficult airway. Anesthesiology 1989; 71: 769-778.

10 Walker RW, Ellwood J. The management of difficult intubation in children. Pediatr Anesth 2009; 19: 77-87.

11 Mace SE, Khan N. Needle cricothyrotomy. Emerg Med Clin North Am 2008; 26: 1085-1101, xi.

12 Peak DA, Roy S. Needle cricothyroidotomy revisited. Pediatr Emerg Care 1999; 15: 224-226.

13 Ravussin P, Bayer-Berger M, Monnier P. Percutaneous transtracheal ventilation for laser endoscopic procedures in infants and small children with laryngeal obstruction: report of two cases. Can J Anaesth 1987; 34: 83-86.

14 Depierraz B, Ravussin P, Brossard E et al. Percutaneous transtracheal jet ventilation for paediatric endoscopic laser treatment of laryngeal and subglottic lesions. Can J Anaesth 1994; 41: 1200-1207.

15 Smith RB, Myers EN, Sherman H. Transtracheal ventilation in paediatric patients. Case reports. Br J Anaesth 1974; 46: 313-314.

16 Weiss M, Engelhardt T. Proposal for the management of the unexpected difficult pediatric airway. Pediatr Anesth 2010; 20; 454-464. 


\section{Pediatric Anesthesia}

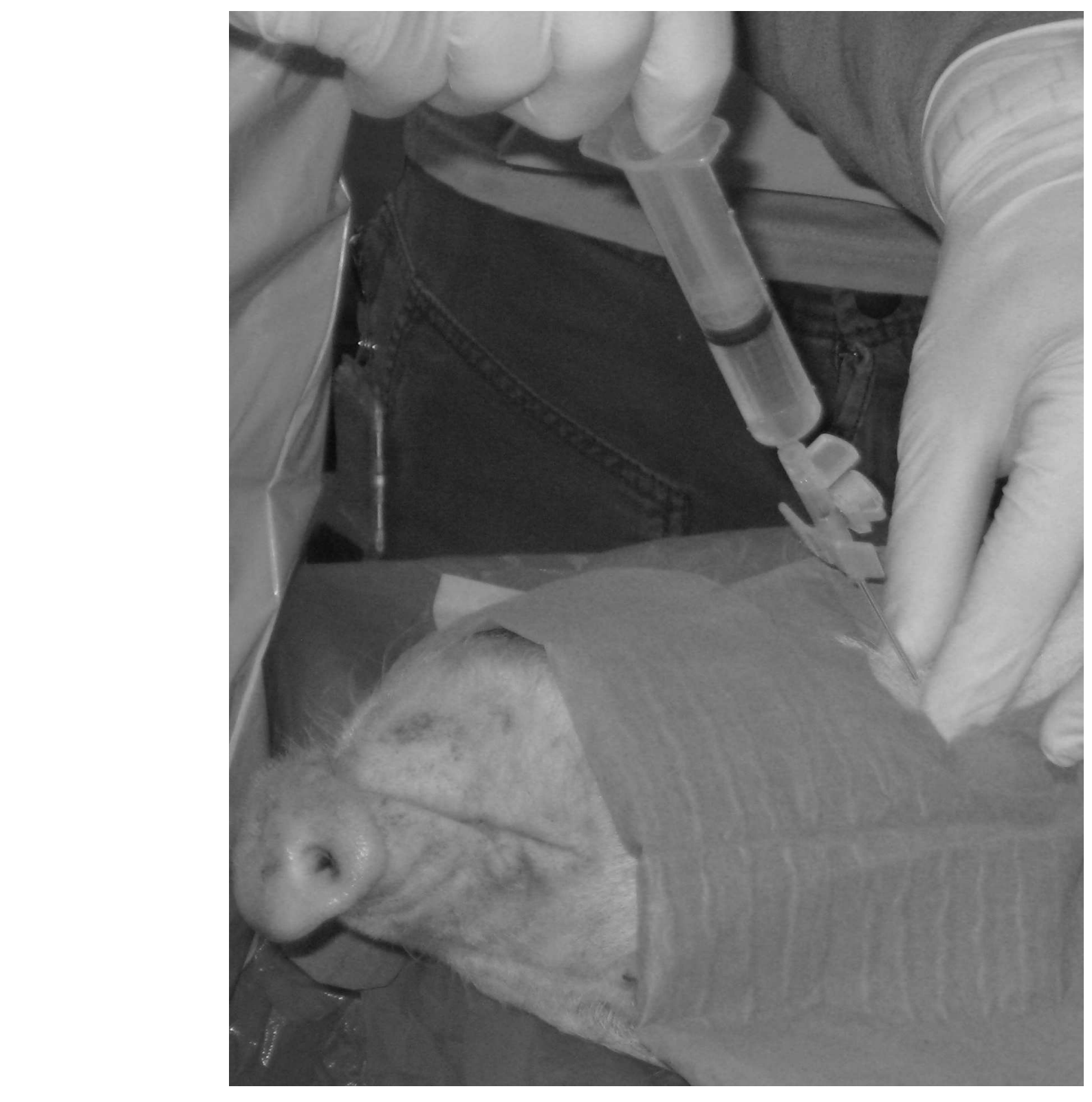

Transtracheal cannula placement.
5

6

7

9

10

11

12

14

15

16

17

19

20

21

23

24

25

26

27

28

30

31

32

33

34

35

36

37

39

40

41

42

43

44

46

47

48

49

50

51

52

53

54

55

56

57

58

59

60

(

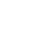

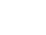

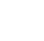

(

(38

(1)

47

48

\section{Page 17 of 24}

2

3

(

\begin{tabular}{l}
10 \\
13 \\
17 \\
18 \\
\hline 19
\end{tabular} $215 \times 269 \mathrm{~mm}(180 \times 180 \mathrm{DPI})$

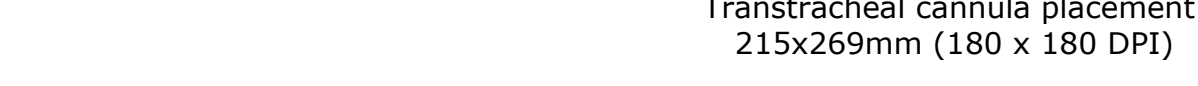




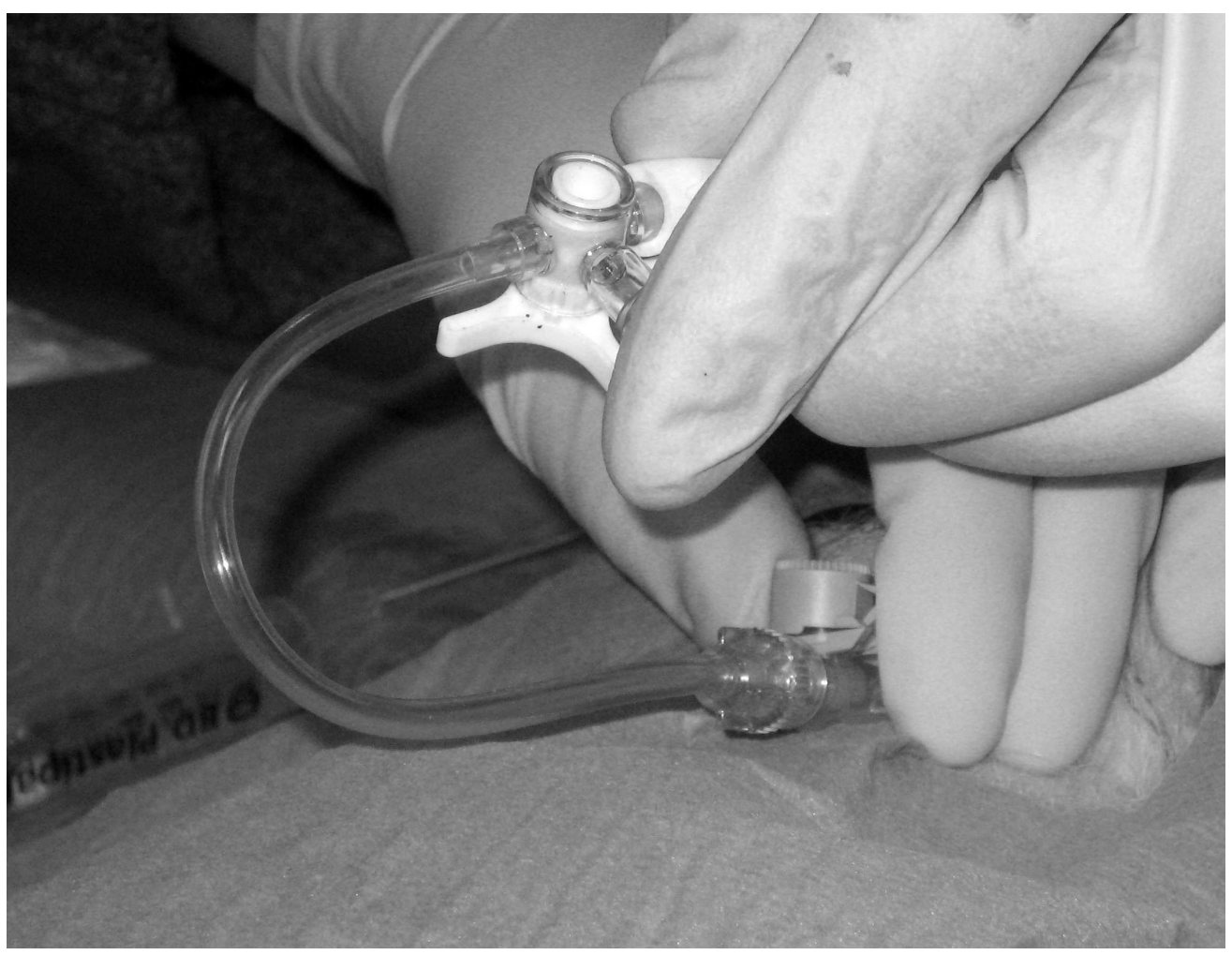

Three-way stopcock used for jet ventilation. $320 \times 245 \mathrm{~mm}(180 \times 180$ DPI $)$ 


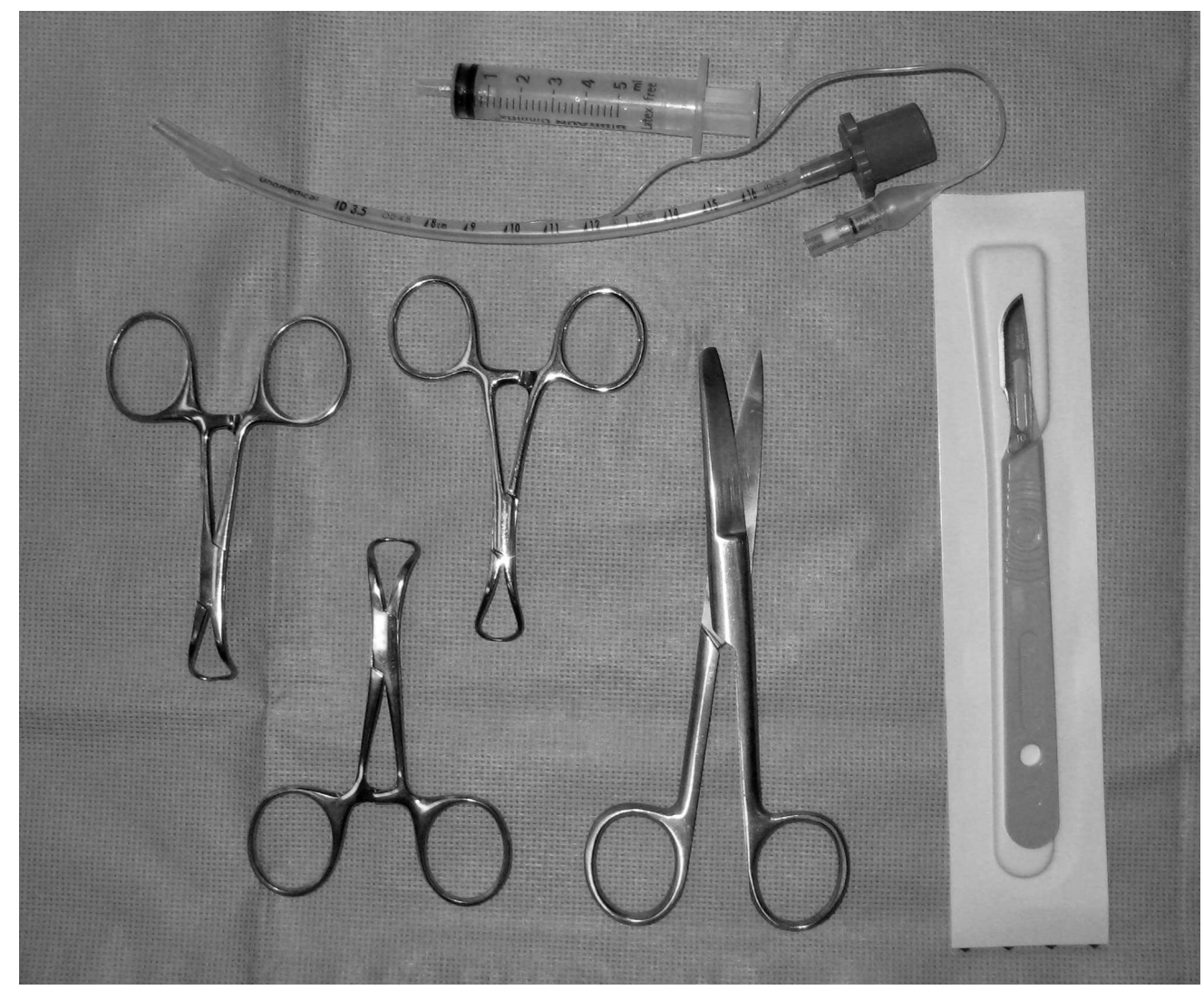

Tools for emergency tracheotomy. Scalpel, nurses scissors, 3 towel forceps. $285 \times 235 \mathrm{~mm}(180 \times 180 \mathrm{DPI})$ 


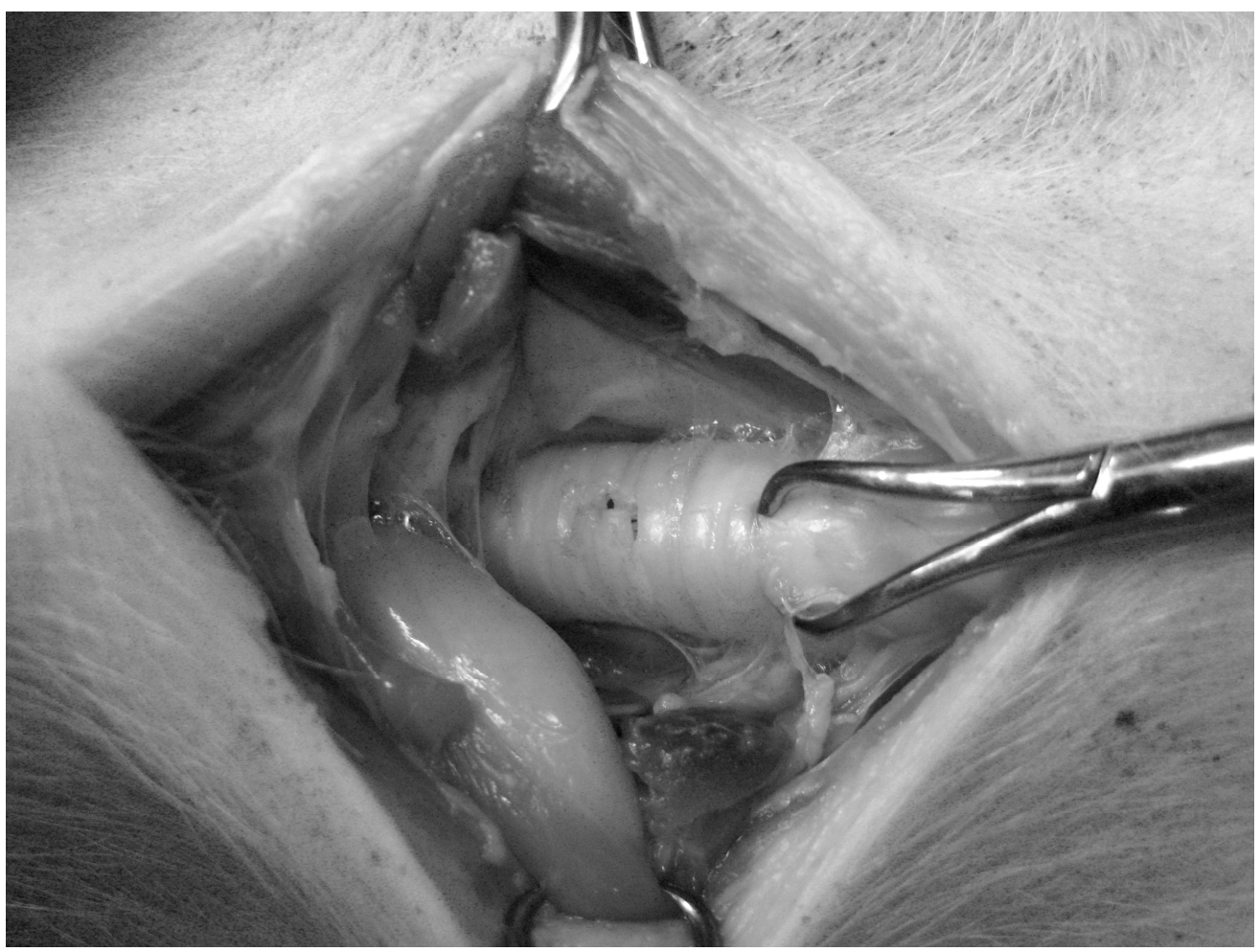

Trachea is presented and stabilised and the strap muscles held aside with three towel forceps. A hole has been cut with in two tracheal rings with the scissors. $433 \times 325 \mathrm{~mm}(180 \times 180 \mathrm{DPI})$ 


\begin{tabular}{|l|c|c|c|}
\hline & $\begin{array}{c}\text { Transtracheal cannula } \\
\mathrm{N}=30\end{array}$ & $\begin{array}{c}\text { Surgical tracheotomy } \\
\mathrm{N}=10\end{array}$ & $\mathrm{P}$ \\
\hline Success & 8 & 8 & 0.007 \\
& $(27 \%, 14-45 \%)$ & $(80 \%, 48-95 \%)$ & \\
\hline Time for successful & $68(35-95)$ & $89(71-200)$ & 0.08 \\
attempt, sec & & & \\
\hline
\end{tabular}


Figure 5. Kaplan-Meier analysis of time and success for performing transtracheal cannula ( $\mathbf{\Lambda})$ and tracheotomy ( $\mathbf{\bullet})$.

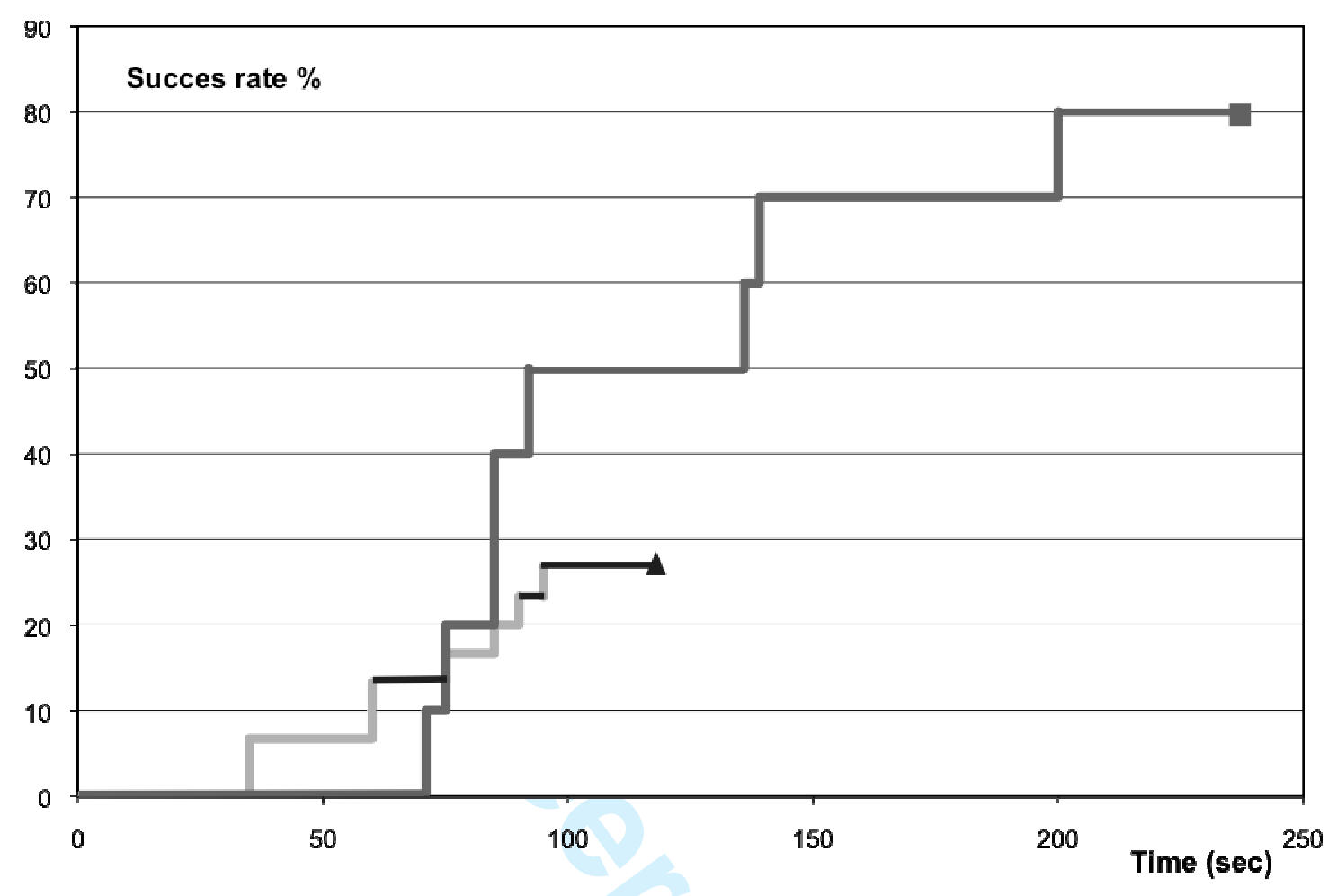


 \\ Page 23 of 24}

Pediatric Anesthesia

17

19




\begin{tabular}{|l|}
\hline Fig 1 \\
Transtracheal cannula placement. \\
\hline Fig 2 \\
Three-way stopcock used for jet ventilation. \\
\hline Fig 3 \\
Tools for emergency tracheotomy. Scalpel, nurses \\
scissors, 3 towel forceps. \\
\hline Fig 4 \\
Trachea is presented and stabilised and the strap \\
muscles held aside with three towel forceps. A \\
hole has been cut with in two tracheal rings with \\
the scissors. \\
\hline
\end{tabular}

Table 1. Time and success rate for obtaining access to the piglet airway, either by insertion of the transtracheal cannula or by performing surgical tracheotomy. 